\title{
Peripheral neuropathy in angioimmunoblastic lymphadenopathy with dysproteinaemia
}

\author{
GIOVA N I TREDICI, MARIO M N A ZZI, A N D \\ EM I L I O LA M P U G N A N I
}

From the Istituto di Anatomia Umana Normale, Università di Milano and Divisione Neurologica,
Ospedale Generale, Vimercate, Milan, Italy

SUMMARY A case of angioimmunoblastic lymphadenopathy with dysproteinaemia complicated by a subacute peripheral neuropathy is described. Clinically the neuropathy was mainly motor, but pain and paraesthesiae in the legs were also present. Sural nerve biopsy indicated decreased numbers of myelinated fibres, mainly the largest, without actual signs of degeneration or regeneration. However, the occurrence of denervation bands indicated that degeneration had led to loss of myelinated fibres.

Angioimmunoblastic lymphadenopathy with dysproteinaemia (AILD) is a rare lymphoma-like disorder which affects the elderly and is characterised clinically by generalised lymphadenopathy, hepatosplenomegaly, and immunological abnormalities (polyclonal gammopathy). The distinctive histological feature is proliferation of small blood vessels and immunoblasts in the lymph nodes, leading to diffuse obliteration of their normal architecture (Frizzera et al., 1974). Angioimmunoblastic lymphadenopathy with dysproteinaemia is not considered a neoplasia, and its similarity with graft versus host reaction has been stressed (Frizzera et al., 1974), although a different opinion has been expressed by Lukes and Tindle (1975), Volk et al. (1975) and Hossfeld et al. (1976).

Peripheral nerve involvement, either direct or indirect, is a well-known complication of the lymphomas (Walsh, 1971a) and other reticuloses (Currie and Henson, 1971). Neuropathies were also reported in monoclonal (Walsh, 1971b) and even in polyclonal gammopathies (McLeod and Walsh, 1975), although much less frequently. However, the association of neuropathy with AILD has not been reported previously.

\section{Case report}

The patient, a 70 year old retired craftsman, was

Address for reprint requests: Dr G. Tredici, Istituto di Anatomia Umana Normale, Università di Milano, Via Mangiagalli 31, 20133 Milano, Italy.

Accepted 16 December 1978 admitted to hospital because of a generalised enlargement of the lymph nodes. He had been well until a month earlier when he began to suffer aching pain and cramps in the legs. On examination (the patient was not seen by a neurologist) only generalised enlargement of lymph nodes, and hepatic edge $20 \mathrm{~mm}$ below the costal margin were found. No neurological signs were reported. Peripheral pulses were present.

All the main biochemical indices, plasma electrolytes, and urine were normal. A blood count showed a mild normochromic anaemia which deteriorated during the next few days and the patient then required blood transfusions. The leucocyte and platelet counts were normal. The direct Coombs test was positive.

Immunoelectrophoresis of the serum proteins disclosed a polyclonal gammopathy. The IgA level was $4.5 \mathrm{~g} / 1$ (normal: $0.5-2$ ), IgG $47 \mathrm{~g} / 1$ (normal: 8-15), IgM $3.45 \mathrm{~g} / 1$ (normal: 0.4-1.2), and they remained raised in repeated estimations throughout the course of the illness.

A lymph node biopsy showed evidence of diffuse obliteration of the parenchymal structure from proliferation of the capillaries and venules with hyperplasia of the endothelium and infiltration by immunoblasts. The histological diagnosis (confirmed by Dr G. Frizzera) was angioimmunoblastic lymphadenopathy with dysproteinaemia. A bone marrow biopsy showed a reduced number of erythroblasts with normal myeloid and platelet series. There was marked erythrophagocytosis and abundant haemosiderin deposits. Plasma cells and 
the immunoblasts were present in normal quantity.

The ECG, ophthalmoscopic examination, and $\mathrm{x}$-ray study of the skull, chest, and pelvis did not show any abnormalities. Mild spondylotic changes were present at the lumbar tract on radiography of the spine. The intersomatic spaces and the spinal canal were of normal width.

\section{COURSE OF ILLNESS}

Paraesthesiae and aching pain in the lower limbs increased and so the patient was treated with high doses of vitamin B12. As there was no improvement, a neurologist was consulted. On examination the lower limb muscles were tender, hypotonic, and weak, especially distally. Hand grip was slightly weak. No sensory abnormalities could be detected, but pain and paraesthesiae were felt spontaneously by the patient in the lower limbs. The abdominal reflexes were elicited with difficulty. The myotatic reflexes of the upper limbs were reduced. Ankle and patellar jerks were absent bilaterally. The Romberg test was negative. There were no cerebellar signs, tremor, or other abnormal involuntary movements.

The CSF was normal. Motor nerve conduction velocity was reduced in the median, ulnar, and lateral popliteal nerves (respectively $45 \mathrm{~m} / \mathrm{s}$, $30 \mathrm{~m} / \mathrm{s}, 38 \mathrm{~m} / \mathrm{s})$. The evoked muscle potentials were less than $0.2 \mathrm{mV}$ in all cases. A sural nerve biopsy was then obtained, before starting any further therapy. The patient was treated with steroids. The enlargement of the lymph nodes decreased, and he was discharged, neurologically unchanged.

Six months later he was readmitted because of the appearance of a maculopapular rash which, at biopsy, was shown to be the result of perivascular lymphocyte infiltrations and haemorrhages in the dermis. The skin papules disappeared spontaneously. On that occasion the patient was reexamined neurologically, and the signs of peripheral nerve involvement were found unchanged.

The patient died 20 months after the disease was discovered from an acute febrile illness. During this period his neurological signs remained unchanged with only mild fluctuation in subjective symptoms.

\section{NECROPSY FINDINGS}

Death was caused by a purulent diffuse leptomeningitis. Macroscopically the lymph nodes were enlarged and the liver and spleen also appeared to be enlarged. Except for a marked hypertrophy of the left ventricle of the heart and pulmonary emphysema, the viscera were normal.

The lymph nodes and the spleen showed a more extensive immunoblastic proliferation and an increased number of mature plasma cells compared with the biopsy specimen. Infiltrations of immunoblasts and plasma cells were observed in the meninges and in the cerebral substance. None of the visceral organs showed cellular infiltrations. The peripheral nerve trunks were not examined histologically.

\section{SURAL NERVE BIOPSY}

The sural nerve was biopsied, prepared, and studied according to previously reported methods (Tredici and Minazzi, 1975). Light microscopy and low power electronmicrographs showed marked reduction of the number of myelinated fibres. Most of the remaining fibres looked normal (Fig. 1) except for a few fibres which showed a doubled myelin sheath and a small axon diameter compared to the thickness of the myelin sheath. However, these are nonspecific changes.

In longitudinal sections the myelin thickness was uniform throughout the length of the internode. All nodes of Ranvier studied were normal, and did not show signs of paranodal delamination of the myelin. The Schwann cells of the myelin- $\triangle$ ated and unmyelinated fibres were normal. The unmyelinated axons had normal distribution, disposition, size, and shape.

There were no specific signs of degeneration, either of the Wallerian type or of segmental demyelination, and no signs of regeneration were observed. The occurrence of some denervation bands of irregular shape (Fig. 2), sometimes containing myelin debris (denervation bands of the myelinated type (Ochoa and Mair, 1969)), provided evidence that previous degeneration had occurred. No clusters of fibres or onion bulb formations were ever observed.

Fibroblasts and blood vessels were normal. No amyloid deposits or foreign cell infiltrates were seen.

The number of myelinated fibres was 4220 per $\mathrm{mm}^{2}$ (normal: 7713-14 373 per $\mathrm{mm}^{2}$ (Dyck et al., 1972)), while the density of the unmyelinated fibres was in the normal range, $34705 \mathrm{~mm}^{2}$ (normal: 32 179-68 813 per $\mathrm{mm}^{2}$ ). The frequency distribution of the diameter of the unmyelinated fibres was normal, while the histogram (Fig. 3) of the myelinated fibres showed a shift to the left of the peak of the large fibres and a decrease in their incidence.

The percentages of the endoneural area covered in cross-sections by the different nerve components are reported in the Table. These data 


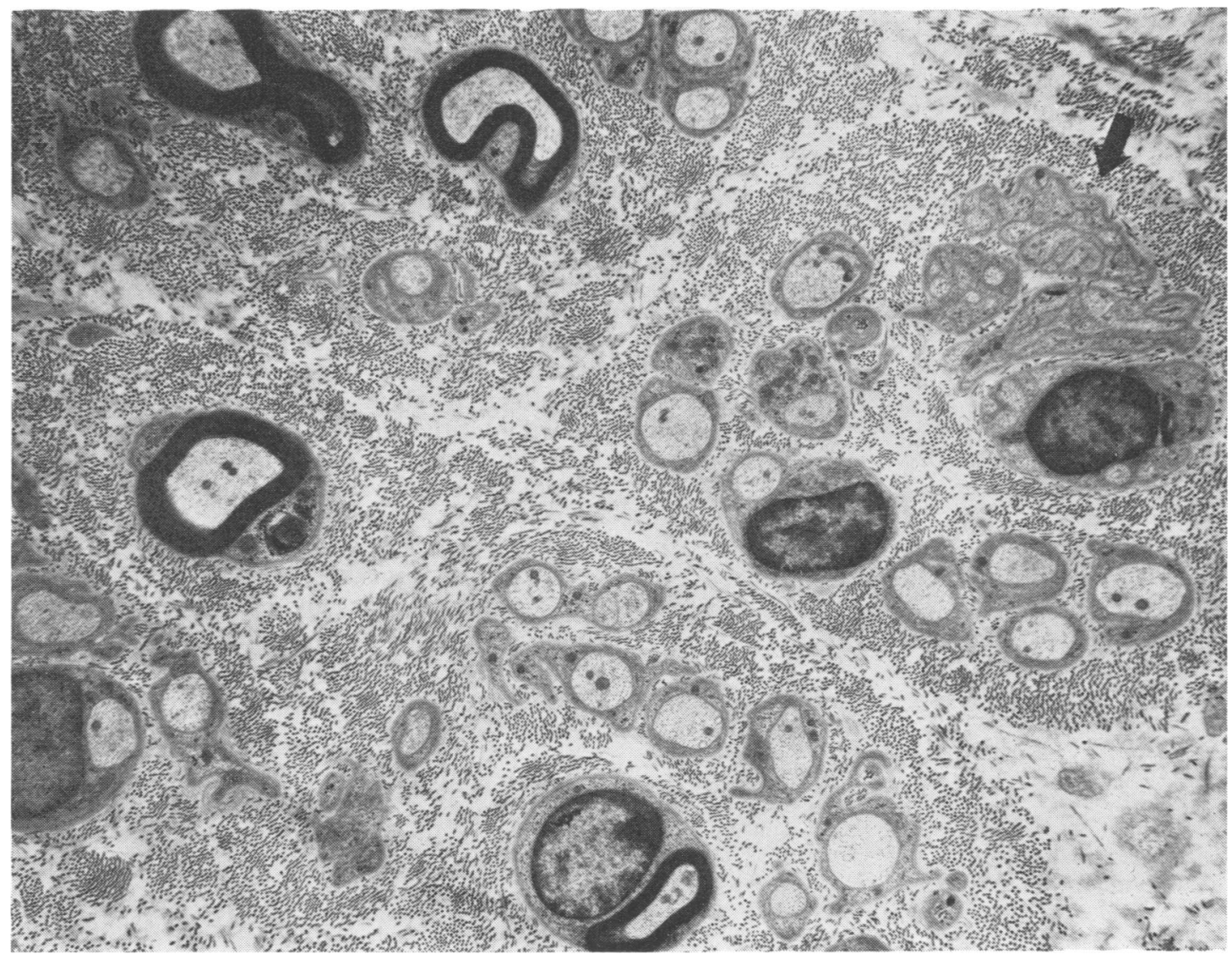

Fig. 1 Low power electron micrograph. Myelinated fibres are reduced in number but normal. There are many normal unmyelinated fibres and a denervation band (arrow). $\times 6000$.

confirmed the marked reduction in density of the myelinated fibres and gave evidence (not apparent in qualitative estimation) of a mild reduction of the area pertinent to the unmyelinated axons.

\section{Discussion}

The present case shows all the typical clinical and histopathological features from which Frizzera et al. (1974) defined AILD. However, our patient also presented a peripheral neuropathy with subacute onset, mainly motor, although pain in the lower limbs was the presenting symptom. The normal serum, urine, and CSF findings excluded a concomitant toxic, metabolic, or postinfective neuropathy. The peripheral nerve involvement is, therefore, to be ascribed in our patient to the concomitant systemic disease.

Association of AILD with peripheral neuropathy has not been reported previously. The nerve had peculiar histological features. Pathological changes were practically confined to the myelinated fibres and mainly the largest ones. A similar situation has been reported only rarely in lymphoma neuropathy (Walsh, 1971a), and does not seem to occur in most of the common toxic, nutritional, and metabolic neuropathies (Thomas et al., 1971; Tredici and Minazzi, 1975; Behse et al., 1977) where all sizes of fibres are affected, although relatively greater damage and loss of large diameter fibres is common.

Another striking feature of AILD neuropathy was the lack of any intermediate stage of either degenerative or regenerative processes. This makes it difficult to assess the type of degenerative changes the nerve had undergone. However, the occurrence of denervation bands of the myelinated type, the absence of demyelinated internodes, and the normal aspects of the Ranvier nodes point to a degeneration of Wallerian type. 


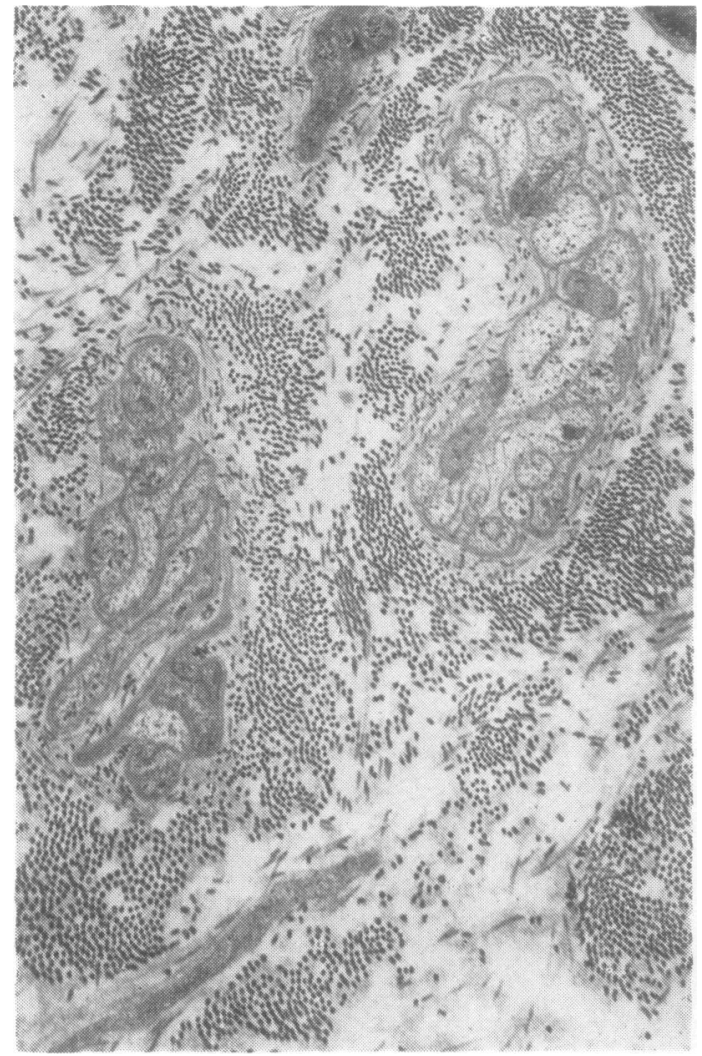

Fig. 2 Two denervation bands of irregular shape. $\times 6000$.
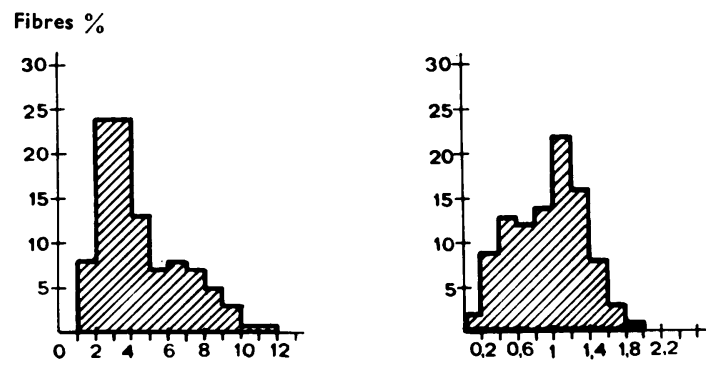

Fig. 3 Frequency distribution of the diameter $(\mu \mathrm{m})$ of myelinated (left) and unmyelinated fibres (right).

Table Percentage of endoneural area covered in cross-sections by different nerve components

\begin{tabular}{lll}
\hline Nerve components & $\begin{array}{l}\text { AILD neuropathy } \\
(70 \text { yr })\end{array}$ & $\begin{array}{l}\text { Control } \\
(52 \text { yr })\end{array}$ \\
\hline Connective tissue & 80 & 47 \\
Myelinated axons & 2.5 & 12 \\
Myelin & 4.5 & 25 \\
Myelinated Schwann cells & 1.5 & 4.5 \\
Unmyelinated axons & 3 & 3.5 \\
Unmyelinated Schwann cells & 9 & 8 \\
\hline
\end{tabular}

The reduced density of myelinated fibres and the decreased percentage of the endoneural area covered by myelinated axons are also in keeping with this view.

In interpreting the pathological changes of the nerve, one must consider the patient's age, since denervation and other pathological changes have been shown to occur in the nerves of the normal elderly (Ochoa and Mair, 1969). The quantitative determinations, giving objective evidence of a markedly decreased density of the myelinated fibres and of the endoneural area pertinent to these fibres, permit rejection of this possibility. On the other hand, aging may explain the mild reduction of the unmyelinated axon component.

In the present case, as in most cases of peripheral neuropathy, it is difficult to assess the mechanism of peripheral nerve lesions. Neuropathy has been reported in lymphoma and other reticuloses, plasmocytoma, and even in association with polyclonal gammopathy, but how these conditions cause nerve lesions is still unclear (McLeod and Walsh, 1975). However, all of these conditions are malignancies, whereas AILD is ${ }^{\circ} \cdot{ }^{\circ}$ considered a hyperimmune state similar in many $\underset{D}{\vec{D}} \vec{c}$ respects to the host versus graft reaction (Frizzera et al., 1974). On the other hand, Lukes and Tindle (1975) reported its potential transformation into malignant immunoblastic sarcoma, and Volk et al. (1975) and Hossfeld et al. (1976) found chromosomal abnormalities in the lymphatic tissue of patients with AILD similar to those found in the lymphomas.

Finally, we would like to stress the agreement in the present case of pathological, clinical, and electrophysiological findings. The selective involvement of the largest myelinated fibres is in accordance with the reduction of nerve conduction velocity (Gilliatt, 1966; Thomas, 1971) and with the prominent motor involvement, provided that the pathological aspects in the sural nerve are representative of the changes in other parts of the peripheral nervous system. The pain experienced by the patient may also be explained by selective involvement of the largest fibres according to Melzack and Wall's (1965) gate-control theory of pain. This theory has been challenged by many authors for lack of clinicopathological correlations in many cases of human peripheral neuropathy (Thomas, 1975). Nevertheless, as stressed by Nathan (1976), discarding this theory must not make us reject the well-established concept that large afferent fibres exert an inhibitory action on the small slower conducting fibres (some of which are concerned with pain), although the site and manner of action in the 
central nervous system are still a matter of conjecture.

We are grateful to Professor C. Schmid for the lymph node, skin, bone marrow biopsy, and necropsy studies, and to our colleagues in the Divisione Medicina I, Ospedale Generale di Vimercate, for permitting us to report the case.

\section{References}

Behse, F., Buchthal, F., and Carlsen, F. (1977). Nerve biopsy and conduction studies in diabetic neuropathy. Journal of Neurology, Neurosurgery, and Psychiatry, 40, 1072-1082.

Currie, S., and Henson, R. A. (1971). Neurological syndromes in the reticuloses. Brain, 94, 307-320.

Dyck, P. J., Lambert, E. H., and Nichols, P. C. (1972). Quantitative measurement of sensation related to compound action potential and number and size of myelinated and unmyelinated fibers of sural nerve in health, Friedreich's ataxia, hereditary sensory neuropathy and tabes dorsalis. In Handbook of Electroencephalography and Clinical Neurophysiology, vol. 9, pp. 83-118. Edited by A. Rémond, Elsevier: Amsterdam.

Frizzera, G., Moran, E. M., and Rappaport, H. (1974). Angio-immunoblastic lymphadenopathy with dysproteinaemia. Lancet, 1, 1070-1073.

Gilliatt, R. W. (1966). Nerve conduction in human and experimental neuropathies. Proceedings of the Royal Society of Medicine, 59, 989-993.

Hossfeld, D. K., Höffen, K., and Schmidt, C. G. (1976.) Chromosome abnormalities in angioimmunoblastic lymphadenopathy. Lancet, 1, 198.

Lukes, R. J., and Tindle, B. H. (1975). Immunoblastic lymphadenopathy: a hyperimmune entity resembling Hodgkin's disease. New England Journal of Medicine, 292, 1-8.

McLeod, J. G., and Walsh, J. C. (1975). Neuropathies associated with paraproteinemias and dysproteinemias. In Peripheral Neuropathy, pp. 1012-1029. Edited by P. J. Dyck, P. K. Thomas, and E. H. Lambert. W. B. Saunders: Philadelphia.

Melzack, M., and Wall, P. D. (1965). Pain mechanisms: a new theory. Science, 150, 971-979.

Nathan, P. W. (1976). The gate-control theory of pain. A critical review. Brain, 99, 123-158.

Ochoa, J., and Mair, W. G. P. (1969). The normal sural nerve in man. Part 2. Changes in the axons and Schwann cells due to ageing. Acta Neuropathologica (Berlin), 13, 217-239.

Thomas, P. K. (1971). The morphological basis for alterations in nerve conduction in peripheral neuropathy. Proceedings of the Royal Society of Medicine, 64, 295-298.

Thomas, P. K., Hollinrake, K., Lascelles, R. G., O'Sullivan, D. J., Baillod, R. A., Moorhead, J. F., and Mackenzie, J. C. (1971). The polyneuropathy of chronic renal failure. Brain, 94, 761-780.

Tredici, G., and Minazzi, M. (1975). Alcoholic neuropathy. An electron-microscopic study. Journal of the Neurological Sciences, 25, 333-346.

Volk, S. L. R., Monteleone, P. L., and Knight, W. A. K. (1975). Chromosomes in AILD. New England Journal of Medicine, 292, 975.

Walsh, J. C. (1971a). Neuropathy associated with lymphoma. Journal of Neurology, Neurosurgery, and Psychiatry, 34, 42-50.

Walsh, J. C. (1971b). The neuropathy of multiple myeloma. An electrophysiological and histological study. Archives of Neurology (Chicago), 25, 404. 414. 